\title{
MODERATE DEVIATIONS FOR BOUNDED SUBSEQUENCES
}

\author{
GEORGE STOICA
}

Received 8 December 2005; Revised 14 April 2006; Accepted 21 April 2006

We study Davis' series of moderate deviations probabilities for $L^{p}$-bounded sequences of random variables $(p>2)$. A certain subseries therein is convergent for the same range of parameters as in the case of martingale difference or i.i.d. sequences.

Copyright (c) 2006 George Stoica. This is an open access article distributed under the Creative Commons Attribution License, which permits unrestricted use, distribution, and reproduction in any medium, provided the original work is properly cited.

\section{Introduction and main results}

Let $\left(X_{n}\right)_{n \geq 1}$ be a sequence of random variables on a probability space $(\Omega, \mathscr{F}, P)$ and $p \geq 1$ a fixed real number. We say that $\left(X_{n}\right)_{n \geq 1}$ is $L^{p}$-bounded if it has finite $p$ th moments, that is, $\left\|X_{n}\right\|_{p} \leq C$ for some $C>0$ and any $n \geq 1$. Let $\varepsilon>0$; finding the rate of convergence of the moderate deviations probabilities $P\left[\left|\sum_{k=1}^{n} X_{k}\right|>\varepsilon a_{n}\right]$ with $a_{n}=(n \log n)^{1 / 2}$ or $(n \log \log n)^{1 / 2}$ is known in the literature as Davis' problems. More precisely, let $\delta=$ $\delta(p) \geq 0$ be a function of $p \geq 1$ and consider the series

$$
\begin{array}{r}
\sum_{n=2}^{\infty} \frac{(\log n)^{\delta}}{n} P\left[\left|\sum_{k=1}^{n} X_{k}\right|>\varepsilon(n \log n)^{1 / 2}\right], \\
\sum_{n=3}^{\infty} \frac{1}{n(\log n)^{\delta}} P\left[\left|\sum_{k=1}^{n} X_{k}\right|>\varepsilon(n \log \log n)^{1 / 2}\right] ;
\end{array}
$$

the convergence of series (2.1) has been studied by Davis (see [7, 8]) and Gut (see [10]) when $\left(X_{n}\right)_{n \geq 1}$ are $L^{P}$-bounded i.i.d. sequences, and by Stoica (see $\left.[14,15]\right)$ when $\left(X_{n}\right)_{n \geq 1}$ are $L^{p}$-bounded martingale difference sequences.

In the sequel, we are interested in Davis' theorems under the only assumption that $\left(X_{n}\right)_{n \geq 1}$ is $L^{p}$-bounded. Our results rely on the "subsequence principle," that is, given any sequence of $L^{p}$-bounded random variables, one can find a subsequence that satisfies, together with all its further subsequences, the same type of limit laws as do i.i.d. variables (or martingale difference sequences) with similar moment bounds. This principle 
was introduced by Chatterji (see [4-6]) and unifies results by Banach and Saks, Komlòs, Révész, Steinhaus in the context of law of large numbers, iterated logarithm, and central limit theorem; extensions to exchangeable sequences were given by Aldous [1] and Berkes and Péter [3]. Also note that Gut [11] and Asmussen and Kurtz [2] gave necessary and sufficient requirements for subsequences to satisfy the famous Hsu- Robbins-Erdős complete convergence result related to the law of large numbers. Our results go a step further, that is, we replace the i.i.d. assumption by $L^{p}$-boundedness, and consider Davis normalizing factors $\sum_{n=2}^{\infty}(\log n)^{\delta} / n$ and $\sum_{n=3}^{\infty}\left(1 / n(\log n)^{\delta}\right)$ instead of complete convergence. We thus have the following.

Theorem 1.1. For any $p>2$ and $L^{p}$-bounded sequence $\left(X_{n}\right)_{n \geq 1}$, there exist $1 \leq n_{1}<n_{2} \cdots$ such that the series

$$
\sum_{N=2}^{\infty} \frac{(\log N)^{\delta}}{N} P\left[\left|\sum_{k=1}^{N} X_{n_{k}}\right|>\varepsilon(N \log N)^{1 / 2}\right]
$$

is convergent for any $0 \leq \delta<p / 2-1$ and any $\varepsilon>0$.

Theorem 1.2. For any $p \geq 2$ and $L^{p}$-bounded sequence $\left(X_{n}\right)_{n \geq 1}$, there exist $1 \leq n_{1}<n_{2} \cdots$ such that the series

$$
\sum_{N=3}^{\infty} \frac{1}{N(\log N)^{\delta}} P\left[\left|\sum_{k=1}^{N} X_{n_{k}}\right|>\varepsilon(N \log \log N)^{1 / 2}\right]
$$

is convergent for any $\varepsilon>0$ if either $\delta>1$, or $\delta=1$ and $p>2$.

If $\delta=1$, Theorem 1.1 holds under the same hypotheses (i.e., $p>4$ ), as in the case of martingale difference sequences (see [15]). In the i.i.d case, Theorem 1.1 holds for $L^{2}$ bounded centered sequences (see $[7,10]$ ).

Theorem 1.2 holds under the same hypotheses as in the case of martingale difference sequences (see [14]). In the i.i.d. case, slightly less than a second moment is needed: $E\left[X_{n}^{2} \log ^{+} \log ^{+}\left|X_{n}\right|^{-\eta}\right]<\infty$ for some $0<\eta<1$ (see $[8,10]$ ), and for necessary moment conditions, one may consult [13].

In the case of martingale difference sequences, Theorem 1.1 fails if $\delta \geq p / 2-1$ and Theorem 1.2 fails if $0 \leq \delta<1$ (see [14]), therefore Theorems 1.1 and 1.2 are the best results one can expect in the $L^{p}$-bounded case.

\section{Proofs}

Proof of Theorem 1.1. In the sequel we will make use of the so-called $c_{r}$-inequality (see $[12$, page 57$])$, which says that

$$
E|X+Y|^{p} \leq 2^{p-1}\left(E|X|^{p}+E|Y|^{p}\right)
$$

for any random variables $X, Y$ and $p>1$. Throughout the paper, $C$ denotes a constant that depends on $p$ and $\varepsilon$ (but not on $k, n, N$ ), and may vary from line to line, even within the same line. 
As $\left(X_{n}\right)_{n \geq 1}$ is bounded in $L^{p}$, according to [9, Corollary IV.8.4], it is weakly sequentially compact. Denote by $\left(Y_{n}\right)_{n \geq 1}$ a subsequence of $\left(X_{n}\right)_{n \geq 1}$ that converges weakly in $L^{p}$ to some $Y \in L^{p}$. Subtracting $Y$ from each element of $\left(Y_{n}\right)_{n \geq 1}$, we reduce the problem to a sequence $\left(Y_{n}\right)_{n \geq 1}$ that converges weakly in $L^{p}$ to 0 . Further, for any $n \geq 1$, we choose a simple random variable $Z_{n}$ (i.e., $Z_{n}$ takes only a finite number of distinct values), such that

$$
\left\|Y_{n}-Z_{n}\right\|_{p}<\frac{1}{2^{n}}
$$

Using Markov's inequality and (2.1), one has

$$
\begin{aligned}
& \sum_{N=2}^{\infty} \frac{(\log N)^{\delta}}{N} P\left[\left|\sum_{k=1}^{N} Y_{n_{k}}\right|>\varepsilon(N \log N)^{1 / 2}\right] \\
& \quad \leq \varepsilon^{-p} \sum_{N=2}^{\infty} \frac{(\log N)^{\delta-p / 2}}{N^{1+p / 2}} E\left|\sum_{k=1}^{N} Y_{n_{k}}\right|^{p} \\
& \quad \leq C\left(\sum_{N=2}^{\infty} \frac{(\log N)^{\delta-p / 2}}{N^{1+p / 2}} E\left|\sum_{k=1}^{N} Z_{n_{k}}\right|^{p}+\sum_{N=2}^{\infty} \frac{(\log N)^{\delta-p / 2}}{N^{1+p / 2}} E\left|\sum_{k=1}^{N}\left(Y_{n_{k}}-Z_{n_{k}}\right)\right|^{p}\right) .
\end{aligned}
$$

According to (2.1), we have

$$
E\left|\sum_{k=1}^{N}\left(Y_{n_{k}}-Z_{n_{k}}\right)\right|^{p} \leq 2^{p-1}\left(E\left|Y_{n_{1}}-Z_{n_{1}}\right|^{p}+E\left|\sum_{k=2}^{N}\left(Y_{n_{k}}-Z_{n_{k}}\right)\right|^{p}\right),
$$

whence by iteration

$$
E\left|\sum_{k=1}^{N}\left(Y_{n_{k}}-Z_{n_{k}}\right)\right|^{p} \leq \sum_{k=1}^{N} 2^{k(p-1)} E\left|Y_{n_{k}}-Z_{n_{k}}\right|^{p}
$$

and assumption (2.2) yields

$$
E\left|\sum_{k=1}^{N}\left(Y_{n_{k}}-Z_{n_{k}}\right)\right|^{p} \leq \sum_{k=1}^{N} 2^{k(p-1)-n_{k} p} \leq \sum_{k=1}^{N} \frac{1}{2^{k}} \leq 1 \quad \text { for any } N \geq 1,
$$

(we used that the subsequence $\left(n_{k}\right)_{k \geq 1}$ is strictly increasing, so $n_{k} \geq k$ ), therefore the last series in (2.3) converges. To prove Theorem 1.1, it suffices to exibit a subsequence $\left(n_{k}\right)_{k \geq 1}$ such that

$$
\sum_{N=2}^{\infty} \frac{(\log N)^{\delta-p / 2}}{N^{1+p / 2}} E\left|\sum_{k=1}^{N} Z_{n_{k}}\right|^{p}<\infty
$$

One can see that $\left(Z_{n}\right)_{n \geq 1}$ also converges weakly in $L^{p}$ to 0 . Indeed, for any $Q \in L^{q}$, where $1 / p+1 / q=1$, we have $E\left(Z_{n} Q\right)=E\left(\left(Z_{n}-Y_{n}\right) Q\right)+E\left(Y_{n} Q\right)$, and the first term on the right-hand side tends to 0 by Hölder's inequality and (2.1), while the second term tends to 0 because $Y_{n}$ converges weakly in $L^{p}$ to 0 . 
4 Moderate deviations for bounded subsequences

By induction, one may choose a subsequence of natural numbers $1 \leq n_{1}<n_{2} \cdots$ such that

$$
E\left[Z_{n_{k}} \mid Z_{i}, i \in I\right] \leq \frac{1}{2^{k}} \quad \text { for each } I \subset\left\{n_{1}, \ldots, n_{k-1}\right\},
$$

where $E\left[Z_{n_{k}} \mid Z_{i}, i \in I\right]$ denotes the conditional expectation of $Z_{n_{k}}$ given the $\sigma$-algebra $\sigma\left(Z_{i}, i \in I\right)$ generated by $\left(Z_{i}\right)_{i \in I}$. This can be done because $\sigma\left(Z_{i}, i \in I\right)$ consists of a finite partition of $\Omega$, and as $Z_{n} \rightarrow 0$ weakly in $L^{p}$, we have $\int_{A} Z_{n} d P \rightarrow 0$ for any $A$ in $\sigma\left(Z_{i}, i \in I\right)$.

We now prove that $\left(n_{k}\right)_{k \geq 1}$ is the required subsequence in (2.7). Indeed, one can write

$$
Z_{n_{k}}=V_{k}+W_{k}
$$

where $E\left[V_{k} \mid V_{1}, \ldots, V_{k-1}\right]=0$ and $\left|W_{k}\right| \leq 1 / 2^{k}$. In particular, $\left(V_{k}\right)_{k \geq 1}$ is a martingale difference sequence. Using Minkowski’s inequality, we deduce that

$$
\left(E\left|\sum_{k=1}^{N} Z_{n_{k}}\right|^{p}\right)^{1 / p} \leq\left(E\left|\sum_{k=1}^{N} V_{k}\right|^{p}\right)^{1 / p}+\left(E\left|\sum_{k=1}^{N} W_{k}\right|^{p}\right)^{1 / p}
$$

According to Burkholder and Hölder's inequalities, we have

$$
E\left|\sum_{k=1}^{N} V_{k}\right|^{p} \leq C E\left(\sum_{k=1}^{N} V_{k}^{2}\right)^{p / 2} \leq C N^{p / 2-1} \sum_{k=1}^{N} E\left|V_{k}\right|^{p} \leq C N^{p / 2}
$$

Also,

$$
E\left|\sum_{k=1}^{N} W_{k}\right|^{p} \leq\left(\sum_{k=1}^{N} \frac{1}{2^{k}}\right)^{p} \leq 1 \quad \text { for any } N \geq 1
$$

Using (2.9)-(2.12), we obtain

$$
\sum_{N=2}^{\infty} \frac{(\log N)^{\delta-p / 2}}{N^{1+p / 2}} E\left|\sum_{k=1}^{N} Z_{n_{k}}\right|^{p} \leq C \sum_{N=2}^{\infty} \frac{(\log N)^{\delta-p / 2}}{N^{1+p / 2}}\left(N^{1 / 2}+1\right)^{p} \leq C \sum_{N=2}^{\infty} \frac{(\log N)^{\delta-p / 2}}{N} .
$$

The latter series in (2.13) is convergent if and only if $\delta<p / 2-1$, thus (2.7) holds and Theorem 1.1 is proved.

Proof of Theorem 1.2. With the same notations and method as in the proof of Theorem 1.1 , it suffices to prove the following analog of (2.7):

$$
\sum_{N=3}^{\infty} \frac{1}{N^{1+p / 2}(\log N)^{\delta}(\log \log N)^{p / 2}} E\left|\sum_{k=1}^{N} Z_{n_{k}}\right|^{p}<\infty .
$$

Using (2.9)-(2.12), the series in (2.14) is dominated by

$$
C \sum_{N=3}^{\infty} \frac{\left(N^{1 / 2}+1\right)^{p}}{N^{1+p / 2}(\log N)^{\delta}(\log \log N)^{p / 2}} \leq C \sum_{N=3}^{\infty} \frac{1}{N(\log N)^{\delta}(\log \log N)^{p / 2}} .
$$


The latter series in (2.15) is convergent if and only if either $\delta>1$, or $\delta=1$ and $p>2$; thus (2.14) holds and Theorem 1.2 is proved.

\section{Acknowledgments}

The author is indebted to an unknown referee and an Associate Editor who helped to improve an earlier version of the manuscript.

\section{References}

[1] D. J. Aldous, Limit theorems for subsequences of arbitrarily-dependent sequences of random variables, Zeitschrift fur Wahrscheinlichkeitstheorie und Verwandte Gebiete 40 (1977), no. 1, 5982.

[2] S. Asmussen and T. G. Kurtz, Necessary and sufficient conditions for complete convergence in the law of large numbers, The Annals of Probability 8 (1980), no. 1, 176-182.

[3] I. Berkes and E. Péter, Exchangeable random variables and the subsequence principle, Probability Theory and Related Fields 73 (1986), no. 3, 395-413.

[4] S. D. Chatterji, A general strong law, Inventiones Mathematicae 9 (1970), no. 3, 235-245.

[5] _ A principle of subsequences in probability theory: the central limit theorem, Advances in Mathematics 13 (1974), no. 1, 31-54.

[6] _ A subsequence principle in probability theory. II. The law of the iterated logarithm, Inventiones Mathematicae 25 (1974), no. 3-4, 241-251.

[7] J. A. Davis, Convergence rates for probabilities of moderate deviations, Annals of Mathematical Statistics 39 (1968), 2016-2028.

[8]__ Convergence rates for the law of the iterated logarithm, Annals of Mathematical Statistics 39 (1968), 1479-1485.

[9] N. Dunford and J. T. Schwartz, Linear Operators. Part I. General Theory, Wiley Classics Library, John Wiley \& Sons, New York, 1988.

[10] A. Gut, Convergence rates for probabilities of moderate deviations for sums of random variables with multidimensional indices, The Annals of Probability 8 (1980), no. 2, 298-313.

[11] On complete convergence in the law of large numbers for subsequences, The Annals of Probability 13 (1985), no. 4, 1286-1291.

[12] M. Loève, Probability Theory. I, 4th ed., Graduate Texts in Mathematics, vol. 45, Springer, New York, 1977.

[13] A. Spătaru, On a series concerning moderate deviations, Revue Roumaine de Mathématiques Pures et Appliquées 45 (2000), no. 5, 883-896 (2001).

[14] G. Stoica, Davis-type theorems for martingale difference sequences, Journal of Applied Mathematics and Stochastic Analysis 2005 (2005), no. 2, 159-165.

[15] __ Series of moderate deviation probabilities for martingales, Journal of Mathematical Analysis and Applications 308 (2005), no. 2, 759-763.

George Stoica: Department of Mathematical Sciences, University of New Brunswick, P.O. Box 5050, Saint John, NB, Canada E2L 4L5

E-mail address: stoica@unbsj.ca 


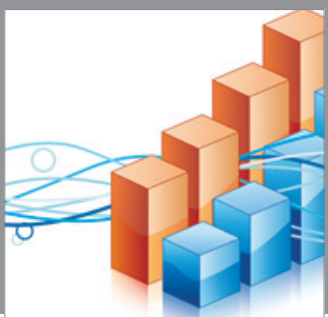

Advances in

Operations Research

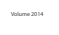

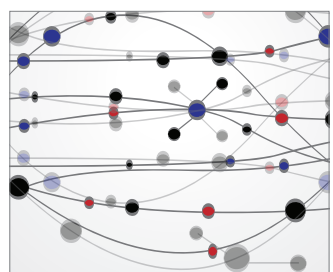

\section{The Scientific} World Journal
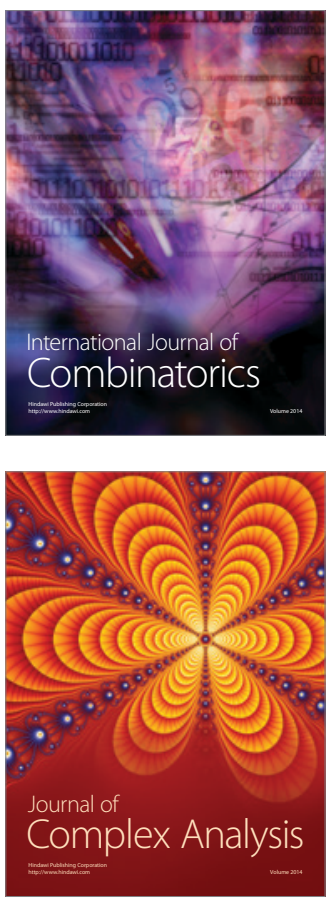

International Journal of

Mathematics and

Mathematical

Sciences
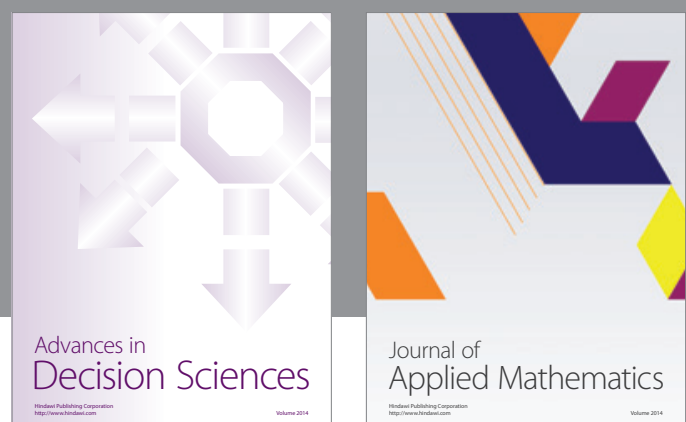

Journal of

Applied Mathematics
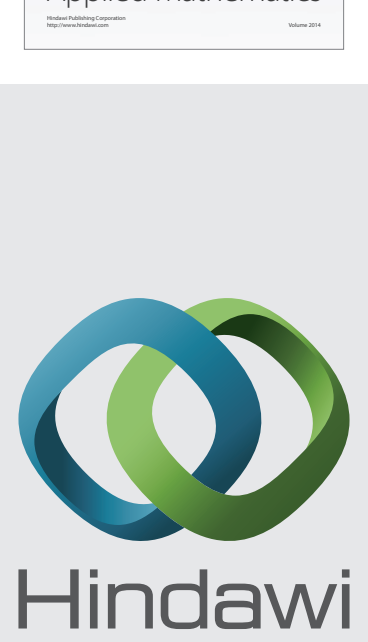

Submit your manuscripts at http://www.hindawi.com
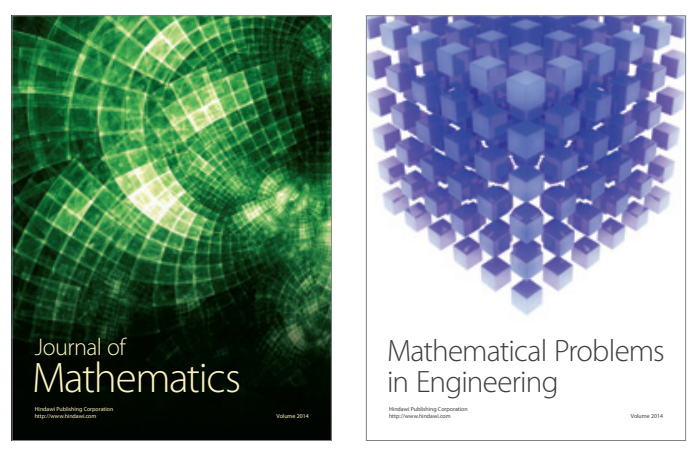

Mathematical Problems in Engineering
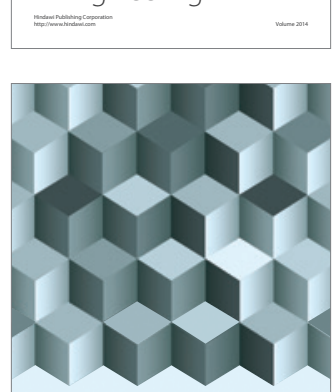

Journal of

Function Spaces
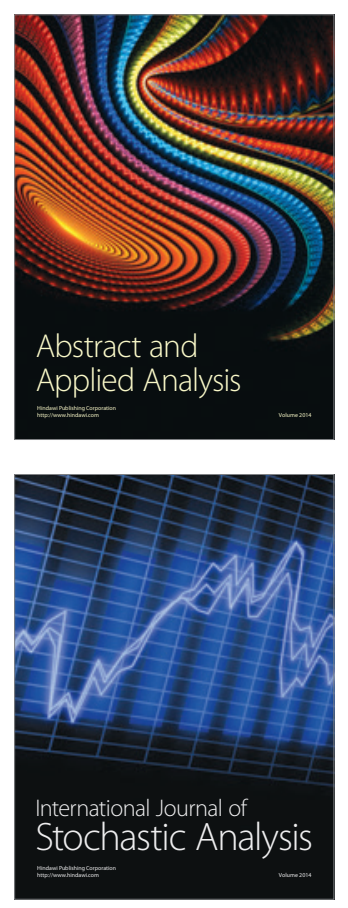

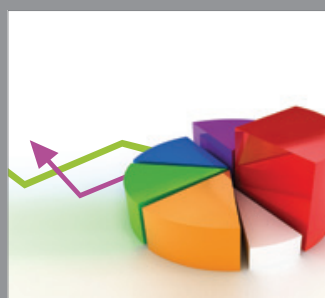

ournal of

Probability and Statistics

Promensencen
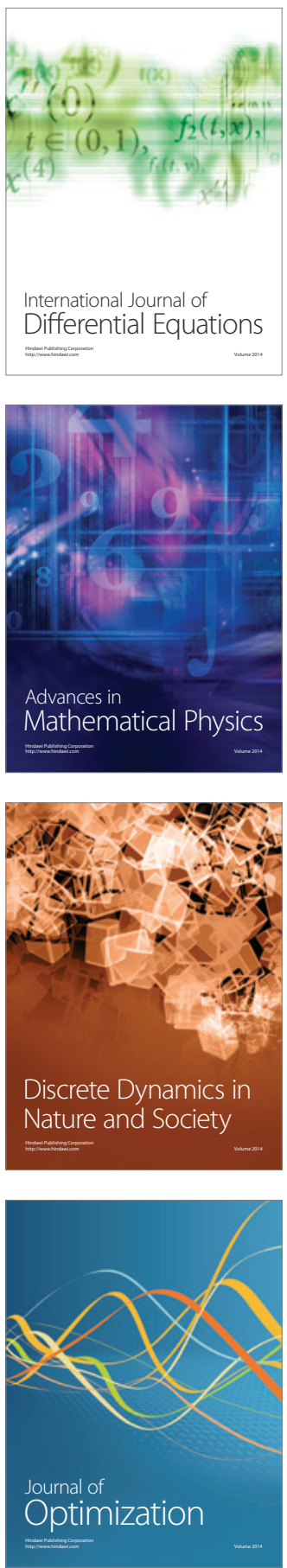